\title{
Differential binding of apo and holo human transferrin to meningococci and co-localisation of the transferrin-binding proteins (TbpA and TbpB)
}

\author{
N. B. L. POWELL, K. BISHOP, H. M. PALMER, D. A. ALA'ALDEEN*, A. R. GORRINGE $\dagger$ \\ and S. P. BORRIELLO $\ddagger$
}

Microbial Pathogenicity Research Group, *Division of Microbiology and Institute of Infections and Immunity, Queen's Medical Centre, Nottingham NG7 2UH,

†Centre for Applied Microbiology and Research, Salisbury, Wiltshire SP4 OJG, and

¥Central Public Health Laboratory, 61 Colindale Avenue, Colindale, London NW9 5HT

\begin{abstract}
Apo-transferrin (apo-hTf) and holo-transferrin (holo-hTf) were separately conjugated to 15-nm colloidal gold. Iron-restricted Neisseria meningitidis strain SD (B:15:P1.16) bound up to three-fold more holo-hTf than apo-hTf $(p<0.001)$. The ability of meningococcal mutants lacking either transferrin-binding protein $A$ (TbpA) or TbpB to discriminate between apo-hTf and holo-hTf was also investigated. There was no significant difference between the amount of gold-labelled apo-transferrin bound by the isogenic TbpA mutant (expressing TbpB) and the parent strain, whereas an isogenic TbpB mutant (expressing TbpA) bound significantly less gold-labelled apo-hTf. The isogenic TbpA and TbpB mutants and the parent strain all bound significantly more holo-hTf than apo-hTf, whereas the double 'knock-out' mutant failed to bind hTf irrespective of the ironloading. In the isogenic mutants, TbpB was more effective in binding either apo- or holo-hTf than TbpA. Monoclonal antibodies against TbpA and TbpB were used to colocalise the transferrin-binding proteins on strain SD. The ratio of TbpA:TbpB was approximately $1: 1$. TbpA and $\mathrm{TbpB}$ were occasionally observed in close proximity to each other, but the two proteins were generally quite separate, which may indicate that they do not usually form a complex to act as a transferrin receptor.
\end{abstract}

\section{Introduction}

Neisseria meningitidis is one of three major causes of bacterial meningitis, and the only organism capable of causing epidemic outbreaks. Additional interest in this pathogen arises because of the high associated mortality following infection and the lack of a fully cross-protective and long-lasting vaccine. In keeping with all bacterial pathogens, $N$. meningitidis must cope with limited free iron when in the human host, estimated to be at about $10^{-8} \mathrm{M}$ [1]. Unlike many bacteria that produce low molecular mass compounds that bind iron with high affinity (siderophores), $N$. meningitidis acquires iron during infection by binding directly to host iron-binding proteins. The most studied of these are the lactoferrin-binding proteins [2] and the transferrin-binding proteins (Tbps) TbpA and TbpB (formerly designated Tbp1 and Tbp2) [3-9]. Attention

Received 23 April 1997; revised version accepted 15 July 1997.

Corresponding author: Professor S. P. Borriello. has focused on the Tbps because of their potential critical role in bacterial survival during infection, and their surface exposure. Both of these factors make these proteins attractive vaccine candidates $[4,10,11]$. Of the two Tbps, TbpB is of most variable molecular mass $(65-90 \mathrm{kDa})$ whereas TbpA is of higher, but less variable $(90-100 \mathrm{kDa})$ molecular mass $[3,7,11,12]$. Strains of meningococci examined to date produce either low molecular mass or high molecular mass Tbp combinations $[3,11,12]$. It is unclear whether meningococci are able to discriminate between apo- and holo-transferrin. Schryvers and Morris [6] used human transferrin (hTf) conjugated to horse radish peroxidase (HRP-hTf) to detect the transferrin receptor in intact meningococci in dot blot assays. They demonstrated that apo-transferrin was less able than $100 \% \mathrm{Fe}$ saturated transferrin to compete with HRP-hTf binding. Increased binding of holo-compared to apo-transferrin has also been reported for $N$. gonorrhoeae $[13,14]$. In contrast, Simonson et al. [8] and Tsai et al. [9] (both groups used an ${ }^{125} \mathrm{I}-\mathrm{TF}$ assay method) concluded that meningococci could not discriminate between apotransferrin and $30 \%$ or $100 \%$ iron-saturated transferrin 
respectively. As it would be advantageous for iron acquisition by meningococci to differentiate between apo- and holo-transferrin, and it has recently been confirmed that $N$. gonorrhoeae has this discriminatory ability [14], the present study aimed to re-examine the question of whether this occurred in meningococci. A previous study, which used gold-labelled transferrin, demonstrated that the transferrin receptor is surface exposed and expressed in vivo [4]. Little is known about the ratio of $\mathrm{TbpA}$ to $\mathrm{TbpB}$ in wild-type strains of meningococci, or their proximity to each other, or their relative contribution in binding transferrin. Furthermore, there is still controversy about the extent to which they act as a single complex.

\section{Materials and methods}

\section{Bacterial strains and growth conditions}

The meningococcal strains used in this study were as follows: strain SD (B:15:P1.16), strain B16B6 (B:2a:P1.2) and the following isogenic mutants of strain B16B6:N16T1E (an isogenic mutant expressing TbpB), N16T2K (an isogenic mutant expressing TbpA) and N16T12EK (a mutant that fails to express either Tbp). These mutants were provided by $\mathrm{Dr} A$. B. Schryvers (University of Calgary, Canada) and are described in detail elsewhere [15]. Bacteria were grown in Mueller-Hinton Broth (Oxoid) to an $\mathrm{OD}_{540}$ of 1.0 , in iron-restricted conditions with $25 \mu \mathrm{M}$ desferrioxamine (Desferal; Ciba) or iron-sufficient conditions with ferric dicitrate $0.5 \% \mathrm{v} / \mathrm{v}$ as described in detail previously [4]. The Escherichia coli strain expressing the cloned TbpA of meningococcal strain SD has been described previously [5].

\section{Sera}

Murine monoclonal antibodies (MAbs) were raised by immunising BALB/c mice with biologically active TbpA or TbpB purified from $N$. meningitidis strain SD, by standard techniques and as described elsewhere [16].

MAb 103 (IgG2a), which was raised against native TbpB derived from strain $\mathrm{SD}$, recognises TbpB but not $\mathrm{TbpA}$ and blocks binding of transferrin to TbpB [16]. The protein concentration of undiluted $\mathrm{MAb} 103$ was $2 \mathrm{mg} / \mathrm{ml}$. MAb 2A2 (IgG2a) was raised to recombinant transferrin-binding protein 1 of strain $\mathrm{SD}$ and recognises native $\mathrm{TbpA}$ but not $\mathrm{TbpB}$ by ELISA. The protein concentration of undiluted $\mathrm{MAb} 2 \mathrm{~A} 2$ was $1.25 \mathrm{mg} / \mathrm{ml}$.

\section{Liquid-phase ELISA}

This assay was performed as described elsewhere [16]. MAb 103.3 specificity was determined by Pintor et al. [16]. Mab 2A2 was tested against purified TbpA and $\mathrm{TbpB}$ in an antigen-mediated ELISA. Both TbpA and
TbpB were coated on an ELISA plate (BioRad) at $0.3 \mu \mathrm{g} /$ well, unreacted sites were blocked with BSA $3 \% /$ PBS/Tween-20 for $1 \mathrm{~h}$. MAb $2 \mathrm{~A} 2$ was added at $100 \mu \mathrm{l} /$ well and incubated for $1 \mathrm{~h}$ at room temperature. The ELISA plate was then washed thoroughly, an alkaline phosphatase polyclonal anti-mouse conjugate was used to detect $\mathrm{MAb} 2 \mathrm{~A} 2$. The reaction was visualised with pNPP substrate ( $\mathrm{p}$-nitrophenyl phosphate).

\section{Preparation of gold-labelled human transferrin (Au-hTf) and labelling of cells with Au-hTf}

Apo-hTf (Sigma) and 100\% Fe saturated holo-hTf (Sigma) were separately conjugated to colloidal gold (Biocell) as described previously [3,4]. The $\mathrm{pH}$ of conjugated gold for both preparations was 6.1 and the minimal amount of either apo- or holo-hTf needed to produce a stable conjugate was added to each preparation, giving a final concentration of hTf in each conjugate of $c .190 \mu \mathrm{g} / \mathrm{ml}(2.4 \mathrm{mM})$. Bacteria were labelled with the Au-hTf and processed for transmission electron microscopy as described previously [4].

\section{Localisation of TbpA and TbpB}

Localisation of bound MAbs (MAb 2A2 or MAb 103) was by detection with neat $(10 \mu \mathrm{g} / \mathrm{ml})$ protein A-gold (Biocell). For single labelling experiments, $500 \mu \mathrm{l}$ of harvested meningococci were centrifuged at $3000 \mathrm{~g}$ for 5 min, washed once in phosphate-buffered saline (PBS), centrifuged again and the pellet was allowed to react with $50 \mu \mathrm{l}$ of the appropriate neat MAbs for $1 \mathrm{~h}$ at $25^{\circ} \mathrm{C}$. The cells were then washed once in PBS, centrifuged at $3000 \mathrm{~g}$ and then mixed with $50 \mu \mathrm{l}$ of neat $15-\mathrm{nm}$ protein A-gold for $1 \mathrm{~h}$ at $25^{\circ} \mathrm{C}$. The suspension was then washed once in PBS before negative staining [4], and examined in a Jeol JEM $100 \mathrm{C}$ transmission electron microscope. For doublelabelling experiments the order of reagents was neat $\mathrm{MAb} 2 \mathrm{~A} 2$ for $1 \mathrm{~h}$, washed once in PBS, neat 5-nm protein A-gold for $1 \mathrm{~h}$, free protein $A(1 \mathrm{mg} / \mathrm{ml}$; Sigma) $30 \mathrm{~min}$ (to block any possible unlabelled $\mathrm{MAb}$ 2A2). The bacteria were washed in PBS and incubated with neat $\mathrm{MAb} 103$ for $1 \mathrm{~h}$ at $25^{\circ} \mathrm{C}$, washed once in PBS and incubated for $1 \mathrm{~h}$ in neat $15-\mathrm{nm}$ protein Agold. The cells were washed once in PBS and processed for electron microscopy as described previously [4]. Due to the length of the incubations, all wash buffers and incubation buffers contained $25 \mu \mathrm{M}$ Desferal for meningococci grown under iron-restricted conditions to maintain iron restriction throughout. The mean labelling density was determined from at least 20 cells.

\section{Statistical analysis}

Statistical analysis of the data was with Minitab 8.21 (Minitab Inc., USA). The raw data were transformed by a square root transformation and a two sample $t$ test 
(95\% confidence limits) was performed on the transformed data.

\section{Results}

\section{Specificity of $M A b s$}

MAb 103.3 specifically recognised TbpB and not TbpA [16]. MAb 2A2 specifically recognised TbpA and not TbpB (TbpA: $\mathrm{A}_{405}=1.026$ SD 0.0896; TbpB: $\mathrm{A}_{405}=0.0242 \mathrm{SD} 0.012$, values adjusted for conjugate only controls).

Comparison of binding of gold-labelled holo-hTf (Au-holo-hTf) and gold-labelled apo-hTf (Au-apohTf) to N. meningitidis

The amount of Au-apo-hTf bound as a function of transferrin concentration is shown in Fig. 1 for both iron-deficient and iron-sufficient grown cells. There was no significant difference $(p=0.13)$ between the iron-deficient bacteria labelled with neat or a 1:1 dilution of Au-apo-hTf. To ensure saturating conditions, neat apo-hTf was used for all experiments.

When grown under iron restriction, strain SD (B:15:P1.16) bound up to $300 \%$ more holo-hTf than apo-hTf $(\mathrm{p}<0.001)$ with means and SEM for 50 cells of 303.8 SEM 29.6 (range 46-500) and 98.5 SEM 13.9 (range 16-300), respectively (Tables 1 and 2). The ability of the isogenic mutants and parent strain (B16B6) to discriminate between Au-holo-hTf and Au-apo-hTf was also assessed (Tables 1 and 2). The parent strain (B16B6) bound $250 \%$ more holo-hTf than apo-hTf $(\mathrm{p}<0.001)$. The TbpB mutant (expressing TbpA) bound significantly less hTf (irrespective of iron-loading) than the parent strain $(\mathrm{p}<0.001)$, but there was no significant difference between the TbpA

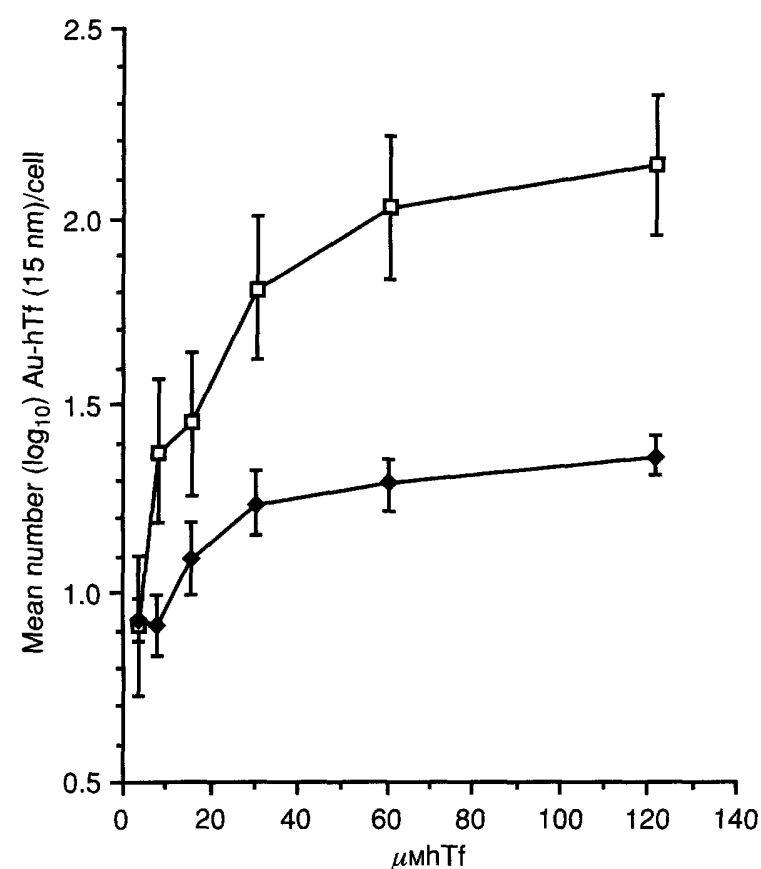

Fig. 1. Isotherm of Au-apo-hTf binding to $N$. meningitidis (strain SD) growth under iron-replete $(\bullet)$ and irondeficient conditions $(\square)$. The plot shows the $\log _{10}$ mean number of $15-\mathrm{nm}$ gold particles (i.e., bound transferrin) per cell and SEM for 20 cells as a function of transferrin concentration.

mutant (expressing $\mathrm{TbpB}$ ) and the parent strain for apo-hTf $(p=0.18)$ or holo-hTf $(p=0.13)$. The double 'knock-out' mutant failed to bind significant amounts of either Au-apo-hTf or Au-holo-hTf compared to the parent strain (both $\mathrm{p}$ values $<0.001$ ).

The Escherichia coli strain expressing TbpA of meningococcal strain SD bound significantly more $(\mathrm{p}=0.0001)$ holo-hTf (mean of 128 SEM 15.4 gold

Table 1. N. meningitidis strain B16B6 (B:2a:P1.2) wild-type and isogenic mutants and strain SD (B:15:P1.16) labelled with $15-\mathrm{nm}$ gold-conjugated neat apo-hTf

\begin{tabular}{|c|c|c|c|c|c|c|}
\hline Strain & $\begin{array}{l}\text { Tbp } \\
\text { expressed }\end{array}$ & $\begin{array}{c}\mathrm{Fe} \\
\text { status }\end{array}$ & $\begin{array}{l}\text { Mean (SEM) number of } \\
\text { gold-labelled transferrin } \\
\text { molecules } / \text { cell }\end{array}$ & Range & $\begin{array}{l}\text { p-value } \\
\text { compared to } \\
\text { parent strain }\end{array}$ & $\begin{array}{l}\text { Percentage } \\
\text { of cells } \\
\text { labelled }\end{array}$ \\
\hline SD & Both & $+\mathrm{Fe}$ & $32.8 \quad(2.1)$ & $20-48$ & $\cdots$ & 100 \\
\hline $\mathrm{SD}$ & Both & $-\mathrm{Fe}$ & $98.5(13.9)$ & $16-300$ & $\ldots$ & 100 \\
\hline B16B6 & Both & $-\mathrm{Fe}$ & $73.5 \quad(9.4)$ & $1-360$ & $\ldots$ & 100 \\
\hline N16T12EK & Neither & $-\mathrm{Fe}$ & $1.9 \quad(0.3)$ & $0-10$ & $<0.001$ & 70 \\
\hline N16T2K & TbpA & $-\mathrm{Fe}$ & $6.0 \quad(0.9)$ & $0-38$ & $<0.001$ & 90 \\
\hline N16T1E & TbpB & $-\mathrm{Fe}$ & $87.6 \quad(9.3)$ & $3-280$ & 0.18 & 100 \\
\hline
\end{tabular}

Table 2. $N$. meningitidis strain B16B6 (B:2a:P1.2) wild-type and isogenic mutants and strain SD (B:15:P1.16) labelled with 15 -nm gold-conjugated neat holo-hTf

\begin{tabular}{|c|c|c|c|c|c|c|}
\hline Strain & $\begin{array}{l}\text { Tbp } \\
\text { expressed }\end{array}$ & $\begin{array}{c}\text { Fe } \\
\text { status }\end{array}$ & $\begin{array}{l}\text { Mean (SEM) number of } \\
\text { gold-labelled transferrin } \\
\text { molecules/cell }\end{array}$ & Range & $\begin{array}{l}\text { p-value } \\
\text { compared to } \\
\text { parent strain }\end{array}$ & $\begin{array}{l}\text { Percentage } \\
\text { of cells } \\
\text { labelled }\end{array}$ \\
\hline SD & Both & $-\mathrm{Fe}$ & $303.8(29.6)$ & $46-500$ & $\ldots$ & 100 \\
\hline B16B6 & Both & $-\mathrm{Fe}$ & $182.6(18.7)$ & $1-500$ & $\ldots$ & 100 \\
\hline N16T12EK & Neither & $-\mathrm{Fe}$ & $2.3 \quad(0.4)$ & $0-15$ & $<0.001$ & 78 \\
\hline N16T2K & TbpA & $-\mathrm{Fe}$ & $15.0 \quad(1.9)$ & $1-60$ & $<0.001$ & 100 \\
\hline N16T1E & TbpB & $-\mathrm{Fe}$ & $131.6(12.8)$ & $0-13$ & 0.13 & 100 \\
\hline
\end{tabular}


particles/cell) than apo-hTf (mean of 63 SEM 8.2 gold particles/cell).

\section{Co-localisation of TbpA and TbpB on the meningococcal cell surface with MAbs}

It was important to ensure that the protein A-gold used to localise the MAb was at saturating levels. This was assessed by allowing strain SD to react with either neat $\mathrm{MAb} 2 \mathrm{~A} 2$ or neat MAb 103, and incubating with various dilutions of protein A-gold (Fig. 2). Both MAb $2 \mathrm{~A} 2$ and MAb 103 were effectively saturated with protein A-gold when neat dilutions were used (1.25 and $2 \mathrm{mg} / \mathrm{ml}$, respectively). For localisation of MAbs, neat protein A-gold $(10 \mu \mathrm{g} / \mathrm{ml})$ was used. The binding of $\mathrm{MAb} 103$ and MAb 2A2 to iron-restricted cells as a function of MAb concentration is shown in Fig. 3. There was no significant difference in the degree of labelling between neat or 1:1 diluted MAb 103 or MAb $2 \mathrm{~A} 2$ ( $\mathrm{p}=0.44$ and 0.34 , respectively). For localisation studies, neat MAbs were used. MAb 103 (IgG2a), which recognises native $\mathrm{TbpB}$ but not native $\mathrm{TbpA}$, was used to determine its ability to detect surfaceexposed epitopes of the TbpB of strain SD (Fig. 4). The $\mathrm{MAb}$ did not react with the $E$. coli-expressed TbpA of strain SD, and failed to label the surface of strain B16B6 or any of its mutants when examined by immunogold electron microscopy. There was significantly $(p<0.0001)$ greater binding of the MAb to meningococcal cells of strain $\mathrm{SD}$ following growth

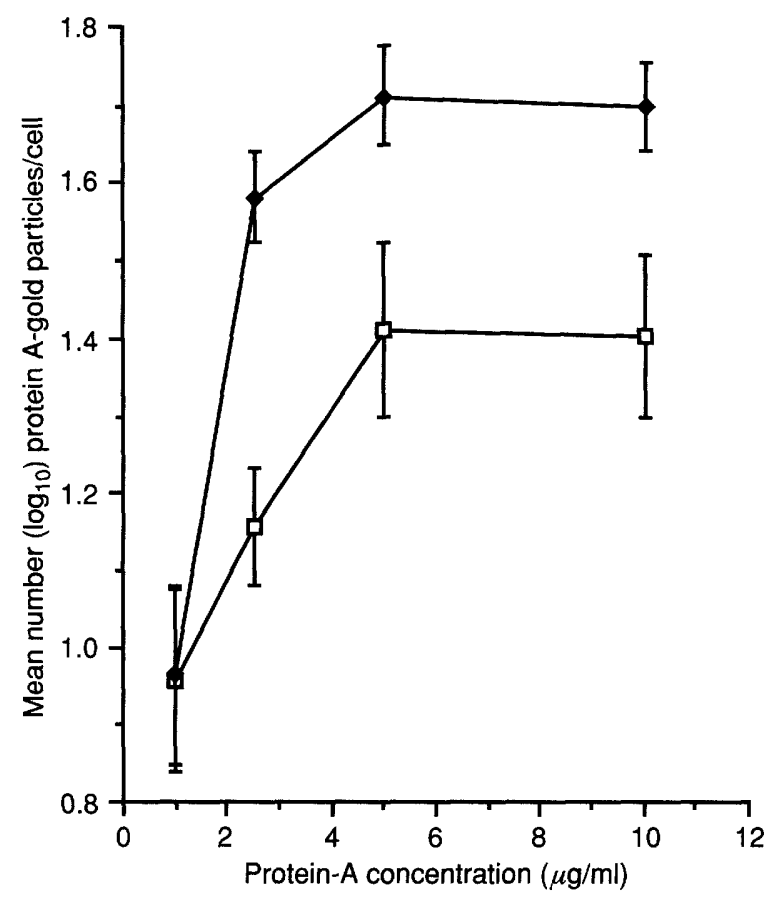

Fig. 2. Effect of varying concentrations of protein A-gold on detection of neat $(2 \mathrm{mg} / \mathrm{ml}) \mathrm{MAb} 103(\bullet)$ and neat $(1.25 \mathrm{mg} / \mathrm{ml}) \mathrm{MAb} 2 \mathrm{~A} 2 \mathrm{(} \square)$ in $N$. meningitidis strain SD grown under iron-deficient conditions. The plot shows the $\log _{10}$ mean number of $15-\mathrm{nm}$ gold-protein A particles per cell and SEM for 20 cells as a function of protein Agold concentration.

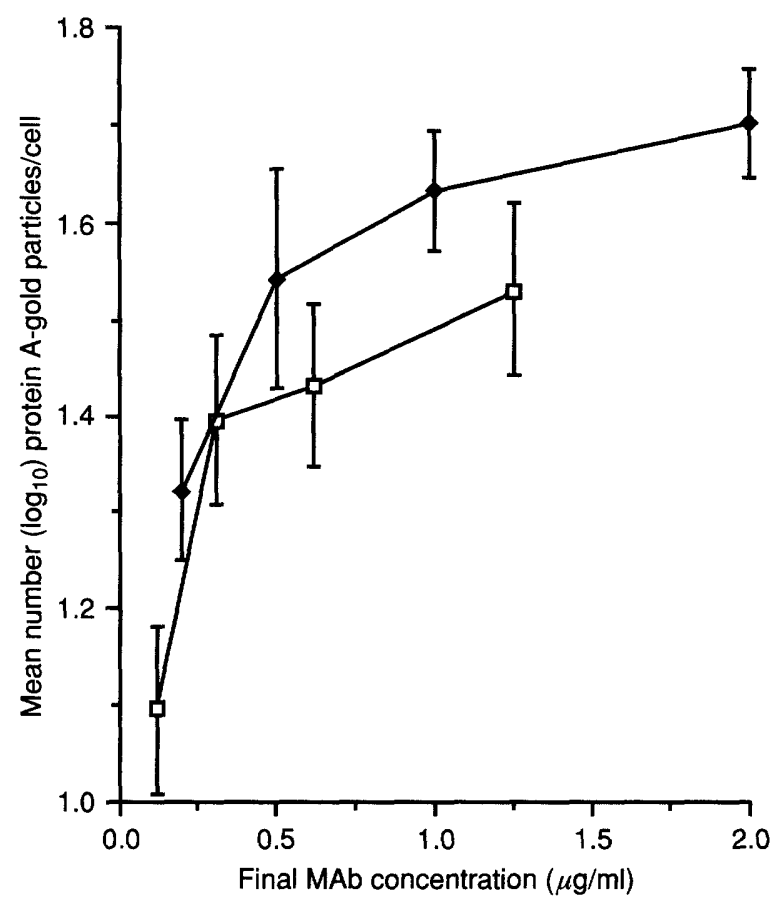

Fig. 3. Isotherm of $\mathrm{MAb} 103(\diamond)$, detecting $\mathrm{TbpB}$, and MAb 2A2 ( $\square$ ), detecting TbpA, with $N$. meningitidis strain SD grown under iron-deficient conditions. The plot shows the $\log _{10}$ mean number of $15-\mathrm{nm}$ gold-protein $\mathrm{A}$ particles per cell and SEM for 20 cells as a function of MAb concentration.

under iron-restricted conditions compared to ironsufficient conditions (Table 3).

$\mathrm{MAb} 2 \mathrm{~A} 2$, which recognises native TbpA but not native $\mathrm{TbpB}$, bound significantly more $(\mathrm{p}=0.0001)$ to strain SD under iron-restricted than iron-sufficient conditions (Table 3). MAb 2A2 and MAb 103 were used to co-localise TbpA and TbpB on strain SD (Fig. 4). Double-labelling with MAb 103 followed by 5-nm protein A-gold and then MAb 2A2 followed by 15-nm protein A gold gave 87.8 SEM 13.5 (range 32240) $15-\mathrm{nm}$ gold particles per cell (MAb 103) and 54.2 SEM 8.66 (range 8-126) 5-nm gold particles per cell (MAb 2A2). The order of labelling was also reversed with $\mathrm{MAb} 2 \mathrm{~A} 2$ followed by $5-\mathrm{nm}$ protein Agold, resulting in 99.8 SEM 11.2 (range 30-240) 5$\mathrm{nm}$ gold particles per cell (MAb 2A2) and 45.6 SEM 6.4 (range 20-120) 15-nm gold particles per cell (MAb 103).

The ratio of TbpA:TbpB could be estimated by comparing 'like size gold' with 'like size gold' in both incubations. The ratio of TbpA:TbpB comparing 5-nm protein A-gold was 99.8 (TbpA): 87.8 (TbpB), giving a ratio of $\mathrm{TbpA}: \mathrm{TbpB}$ of $1.1: 1.0$. Comparing the $15-\mathrm{nm}$ protein A-gold incubations the ratio of TbpA:TbpB was 54.2:45.6, giving a ratio of $\mathrm{TbpA}$ : $\mathrm{TbpB}$ of 1.2:1.0. The double-labelling experiment was done at the same time as incubating the MAbs separately with strain SD (Table 3), allowing statistical comparison of labelling obtained with each MAb used 


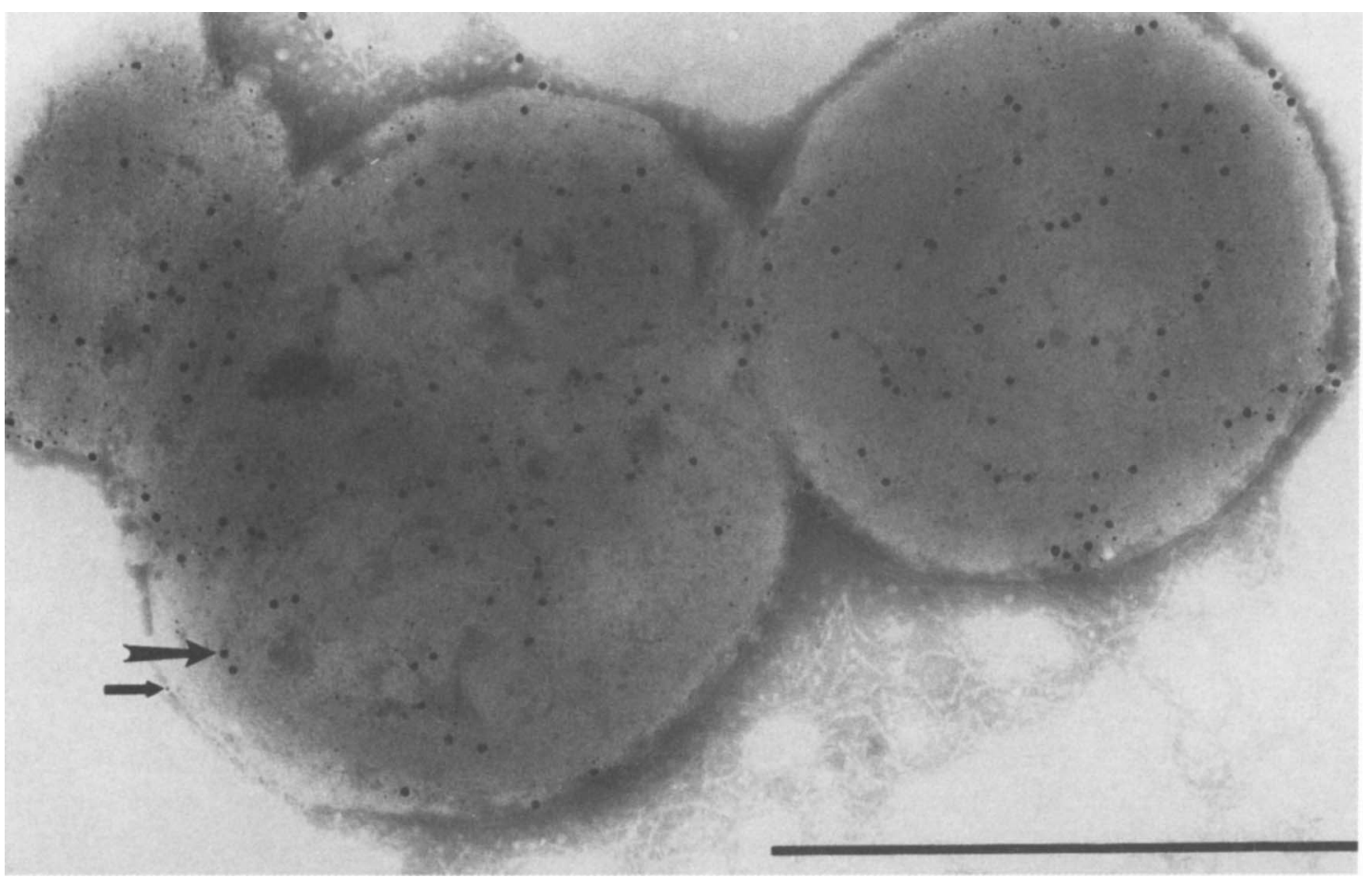

Fig. 4. Co-localisation of TbpA and TbpB on strain SD grown under iron-deficient conditions with MAb 2A2 and 5-nm protein A-gold and MAb 103 and 15-nm protein A-gold respectively.

Table 3. Comparison of total surface-exposed transferrin-binding protein 1 detected by $\mathrm{MAb} 2 \mathrm{~A} 2$ and transferrin-binding protein 2 detected by MAb 103 on strain SD (B:15:P1.16), detected with 15-nm protein Agold

\begin{tabular}{llcc}
\hline Fe status & MAb & $\begin{array}{l}\text { Mean (SEM) number } \\
\text { of gold particles/cell }\end{array}$ & Range \\
\hline$+\mathrm{Fe}$ & MAb103 & $20.9(9.6)$ & $4-200$ \\
$-\mathrm{Fe}$ & MAb103 & $57.7(6.9)$ & $13-120$ \\
$+\mathrm{Fe}$ & MAb2A2 & $9.2(2.5)$ & $0-38$ \\
$-\mathrm{Fe}$ & MAb2A2 & $46.6(8.0)$ & $3-144$ \\
\hline
\end{tabular}

together or singly. Thus it could be determined if steric hindrance would reduce the labelling obtained in the co-localisation experiments. There was no significant difference $(p=0.18)$ between MAb 103 localised by $15-\mathrm{nm}$ protein A-gold alone and the value obtained in the double-labelling experiment. There was also no significant difference for MAb $2 \mathrm{~A} 2(\mathrm{p}=0.13)$.

The co-localisation studies of TbpA and TbpB with MAb 2A2 and MAb 103 demonstrated that most TbpA and TbpB exist separately in strain SD (Fig. 4). A few gold particles were occasionally seen close together (Fig. 4), but these accounted for $<5 \%$ of the gold labelling.

\section{Discussion}

It has been unclear whether meningococci are able to discriminate between apo- and holo-hTf because of the conflicting evidence in the literature. The discrepancy may be due to the different assay methods used (i.e., Tf-horseradish peroxidase and ${ }^{125}$ I-Tf) and different experimental conditions (dead cells and outer-membrane preparations have been used commonly). Simonson et al. [8] and Tsai et al. [9] both concluded that meningococci are unable to discriminate between apoand holo-hTf, although the work of Tsai et al. [9] has been mistakenly cited [17] to the contrary. In contrast, Schryvers and Morris [6] have shown that meningococci can differentiate between apo- and holo-hTf, although the difference in binding was subtle [6]. In keeping with an ability to differentiate, the transferrin receptors in Moraxella catarrhalis [18] and $N$. gonorrhoeae [14] have been conclusively shown to bind holo-hTf in preference to apo-hTf.

The conjugation of gold directly to apo-hTf or holohTf and its use in labelling whole cells can give both an estimation of transferrin receptor numbers and their distribution. Using these methods, this study demonstrated directly and quite clearly that $N$. meningitidis strains B16B6 and SD bind significantly more holohTf than apo-hTf (2.5- and 3-fold respectively). In the Tbp isogenic mutants of strain B16B6, the mutant expressing TbpB bound significantly more $(\mathrm{p}<0.001)$ gold-labelled transferrin (irrespective of iron-loading) than the TbpA-expressing mutant. This is in keeping with the findings of Cornelissen and Sparling for gonococci [14]; they demonstrated that a mutant expressing only TbpA bound significantly less transferrin than the wild-type strain or the strain that expressed only TbpB. In the isogenic mutants of $N$. 
meningitidis strain $\mathrm{B} 16 \mathrm{~B} 6$ both $\mathrm{TbpA}$ and $\mathrm{TbpB}$ preferentially bound holo-hTf $(2.5-$ and 1.5 -fold increase in binding, respectively). The TbpA from strain $\mathrm{SD}$ cloned and expressed in $E$. coli also discriminated between apo- and holo-hTf, binding 2fold more holo-hTf than apo-hTf. This contrasts with the observations in gonococci [14] and $M$. catarrhalis [18] where the ability to differentiate between ferrated and apo-transferrin is restricted primarily to $\mathrm{TbpB}$.

For strain B16B6 the results of analysis of isogenic mutants indicate that the ratio of TbpB:TbpA is almost 15:1 (with apo-hTf) and 9:1 (with holo-hTf), which contrasts strikingly with the ratio of c. 1:1 obtained in the co-localisation study with strain SD. However, it has been demonstrated in gonococci that a mutation in $t b p \mathrm{~B}$ (the gene encoding $\mathrm{TbpB}$ ) has polar effects on $t b p \mathrm{~A}$ (the gene encoding $\mathrm{TbpA}$ ), reducing expression of its associated $\mathrm{TbpA}$ protein. It is likely that this also occurs for meningococci, and as such the true ratio of $\mathrm{TbpB}: \mathrm{TbpA}$ in the parent strain is less than the values obtained when comparing values for each independently determined from isogenic mutants. Unfortunately, the MAbs used for the studies on strain SD do not cross-react with strain B16B6, so a similar approach to look at the ratio on the $\mathrm{B} 16 \mathrm{~B} 6$ parent strain was not possible. It is likely, therefore, that the results from strain $\mathrm{SD}$ are more representative of the natural ratio of TbpB:TbpA in wild-type meningococci. Interestingly, although the ratio of TbpB:TbpA in gonococci determined from isogenic mutants would be expected to be higher than the value in the wild-type parent gonococcal strain, the ratios determined indirectly appear to be the same, i.e., c. 5:1 [14]. Although the ratio of high to low affinity binding sites in the wild-type parent strain may not be reflective of the ratio of $\mathrm{TbpB}: \mathrm{TbpA}$, the observation is still difficult to explain.

Whether or not the TbpB:TbpA ratio is reflective of stoichiometry depends on whether or not the two receptors exist as a functional complex. All published models to date are based on the proposed existence of such a complex $[14,17,19,20]$. However, as yet there is no strong direct evidence for this from cross-linking experiments. Furthermore, the double-labelling experiments presented here failed to demonstrate a proximity between the two Tbps needed for a complex formation. The following evidence has been cited in support of a complex. (1) In neisseriae, mutants expressing only TbpB cannot utilise transferrin as an iron source $[9,21]$, implying a level of interdependence between the two Tbps. (2) Meningococcal mutants expressing only $\mathrm{TbpA}$ cannot grow in media with transferrin as sole iron source [15] and that although corresponding gonococcal mutants can do so, the efficiency of iron uptake from transferrin is decreased [21]. (3) TbpB cannot be affinity purified from a meningococcal mutant lacking TbpA, indicating that the Tbps interact with each other in the wild-type parent strain [15]. (4) The Tbps in gonococci are more susceptible to proteolytic degradation when expressed alone [14]. The inability of meningococcal or gonococcal mutants lacking TbpA to utilise ferrated transferrin as a sole iron source is unquestioned, but it is not necessarily evidence of a complex formation. The ability of gonococci expressing only $\mathrm{TbpA}$ to bind and utilise human transferrin as an iron source to sustain some growth $[21,22]$ is further evidence that a complex of the two Tbps is unnecessary, although the inability of a comparable meningococcal mutant to do this [15] contradicts this conclusion. However, we have shown that this meningococcal mutant (mutant N16T2K of strain B16B6 in this work) expresses very few TbpA transferrin receptors. These observations may explain the absence of any significant growth by this mutant under iron-restricted conditions in the presence of transferrin. The observation that meningococcal $\mathrm{TbpB}$ could not be affinity purified when expressed in the absence of TbpA, has now been shown not to be the case for either meningococci or gonococci when Sarkosyl was replaced with the non-ionic detergent Triton X-100 [14]. This observation does indicate that a cell not expressing TbpA induces a change in characteristics that prevents $\mathrm{TbpB}$ binding to transferrin in Sarkosyl, but not in the presence of other detergents or the absence of detergents. The significance of these observations is difficult to determine, although they do not lead to the conclusion of a complex formation.

The relative susceptibilities of the Tbps of gonococci to proteolytic degradation have been presented as evidence of conformational changes in the Tbps when expressed singly [14]. There is no double that TbpB is more readily degraded in the absence of $\mathrm{TbpA}$ than in its presence [14], implying some direct or indirect change in $\mathrm{TbpB}$, in keeping with the observation of loss of transferrin binding ability in Sarkosyl when expressed alone. An additional conclusion reached by the authors was that TbpA was more susceptible to proteolysis when expressed alone than when expressed with $\mathrm{TbpB}$ in the wild-type strain. However, these conclusions are less secure than those drawn for TbpB. Assessment of remaining TbpB was based on a transferrin-binding bioassay, which would be reflective of only surface-exposed (and protease accessible) components of TbpB, whereas remaining $\mathrm{TbpA}$ was assayed with an antiserum raised to denatured $\mathrm{TbpA}$, which does not recognise surface-exposed epitopes [23]. The surprise, therefore, is not that $\mathrm{TbpA}$ appeared to be resistant to proteolysis, but that there was any detectable degradation at all. In fact the apparent enhanced degradation in the mutant expressing only TbpA could be due to a lower level of TbpA present in the mutant. If this is not the explanation, then the implication is that non-surface-exposed components of TbpA are more readily accessible when expressed 
in the absence of Tbp2, indicating changes in the cell membrane. Overall, there is no doubt that $\mathrm{TbpB}$ behaves differently when expressed in the absence of TbpA, implying that the Tbps do affect each other.

There is evidence that meningococcal Tbps behave cooperatively in wild-type strains. Pintor et al. [24] showed that a polyclonal monospecific antiserum to $\mathrm{TbpB}$ significantly reduced iron uptake from transferrin in homologous wild-type strains. As TbpB does not directly mediate iron uptake, the blocking of transferrin binding to $\mathrm{TbpB}$ must have reduced the capacity of $\mathrm{TbpA}$ to utilise iron from transferrin. The mechanism of this co-operation is unknown. Analysis of transferrin binding data in gonococci is indicative of two binding sites [14]. These sites were considered to be high $(\mathrm{Kd}=0.8 \mathrm{nM})$ and low $(\mathrm{Kd}=16 \mathrm{nM})$ affinity sites of $\mathrm{TbpA}$ and $\mathrm{Tbp} 2$ acting co-operatively. The conclusion that these sites were not TbpA and $\mathrm{TbpB}$ alone was based on the differing $\mathrm{Kd}$ values for these proteins when expressed alone $(\mathrm{Kd}$ for $\mathrm{TbpA}=2.3 \mathrm{nM}$ and for $\mathrm{TbpB}=7.4 \mathrm{nM}$ ). However, these data may be open to mis-interpretation because ferrated transferrin was used in the assays. If the viable gonococci at room temperature used iron from the transferrin during the 20 -min incubation period, then the wild-type strain would have had transferrin affinity values calculated for a mixture of ferrated and apo-transferrin, whereas the TbpB-expressing isogenic mutant would have yielded results for only ferrated transferrin (as $\mathrm{TbpB}$ alone cannot utilise iron from this source). The overall effect in gonococci would be to yield higher $\mathrm{Kd}$ values for $\mathrm{TbpB}$ in the wild-type strain than when expressed alone, due to its higher affinity for ferrated transferrin. Therefore, it remains a possibility that the two sites detected in the wild-type gonococci are separate TbpA and B. The use of apotransferrin in such experiments may overcome the problem of iron utilisation and changing affinities. Although the double-labelling experiments presented here for meningococci rarely demonstrated a proximity between the two Tbps needed for a complex formation, it could be argued that this is due to steric hindrance between the gold particles. It is possible that a protein A-gold particle bound to a $\mathrm{TbpB}$ molecule may block access of the second $\mathrm{MAb}$ or the second protein A-gold particle, or both, to a complexed TbpA molecule. To assess whether steric hindrance was a problem, the MAbs against the Tbps were individually localised on strain SD and the results were compared to the double-labelling experiment. The values obtained in the double-labelling experiment were actually higher (but not significantly different) than those obtained with the MAbs separately. If steric hindrance was a factor to be considered, the labelling would have decreased in the colocalisation study. In the double-labelling experiment, $\mathrm{TbpA}$ and $\mathrm{TbpB}$ were occasionally observed in close proximity to each other (c. 5\%), but generally they were quite separate.
The effectiveness of the double-labelling experiment depended on being able to saturate the appropriate Tbp with the first MAb, and to ensure that the protein A-gold solutions used were saturating. These factors were investigated, and both MAbs and protein A-gold solutions were used neat in order to ensure saturation. Excess free protein A $(1 \mathrm{mg} / \mathrm{ml})$ was used between the first and the second MAbs, to ensure that even if the first MAb was not saturated by the protein A-gold, any unlabelled $\mathrm{MAb}$ would be blocked by the free protein $\mathrm{A}$.

The absence of clear, direct or indirect, evidence of a Tbp complex does not mean that a physical complex does not exist. However, it does strengthen the case for other models to be considered. One such model is as follows. It is possible that $\mathrm{TbpB}$, which can differentiate between apo- and holo-hTf $[14,16,17]$, serves as a receptor to increase the relative concentration of required holo-hTf at the immediate surface environment of the meningococcal cell. The apo-hTf resulting from removal of the iron would be replaced by holo-hTf as a result of the usual lectin-ligand interaction turn-over, and the free apo-hTf removed from the immediate cell-surface environment following interaction with a free $\mathrm{TbpB}$ molecule and its release in favour of holo-hTf. In this way $\mathrm{TbpB}$ may serve to maintain a holo-hTf-rich micro-environment that can be utilised by the cell via TbpA. For such a mechanism to be effective the relative affinities for the Tbps for apo- and ferrated transferrin would have to be in the order of TbpA holo-hTf $>\mathrm{TbpB}$ holo-hTf $>$ TbpB apo-hTf $>$ TbpA apo-hTf. Such relative affinities await experimental confirmation.

This work was funded by a Medical Research Council Programme Grant (no. G9122850). We thank Dr A. B. Schryvers for providing the isogenic mutants and for useful discussions.

\section{References}

1. Bullen JJ, Rogers HJ, Griffiths E. Role of iron in bacterial infection. Curr Top Microbiol Immunol 1978; 80: 1-35.

2. Bonnah RA, Yu R, Schryvers AB. Biochemical analysis of lactoferrin receptors in the Nesseriaceae: identification of a second bacterial lactoferrin receptor protein. Microb Pathog 1995; 19: 285-297.

3. Ala'Aldeen DAA, Davies HA, Wall RA, Borriello SP. The 70 kilodalton iron regulated protein of Neisseria meningitidis is not the human transferrin receptor. FEMS Microbiol Lett 1990; 69: $37-42$.

4. Ala'Aldeen DAA, Powell NBL, Wall RA, Borriello SP Localization of the meningococcal receptors for human transferrin. Infect Immun 1993; 61: 751-759.

5. Palmer HM, Powell NBL, Ala'Aldeen DA, Wilton J, Borriello SP. Neisseria meningitidis transferrin-binding protein 1 expressed in Escherichia coli is surface exposed and binds human transferrin. FEMS Microbiol Lett 1993; 110: 139-146.

6. Schryvers AB, Morris LJ. Identification and characterization of the transferrin receptor from Neisseria meningitidis. $\mathrm{Mol}$ Microbiol 1988; 2: 281-288.

7. Schryvers AB, Lee BC. Comparative analysis of the transferrin and lactoferrin binding proteins in the family Neisseriaceae. Can J Microbiol 1989; 35: 409-415.

8. Simonson C, Brener D, DeVoe IW. Expression of a high- 
affinity mechanism for acquisition of transferrin iron by Neisseria meningitidis. Infect Immun 1982; 36: 107-113.

9. Tsai J, Dyer DW, Sparling PF. Loss of transferrin receptor activity in Neisseria meningitidis correlates with inability to use transferrin as an iron source. Infect Immun 1988; 56: 3132-3138.

10. Ala'Aldeen DA, Stevenson $\mathrm{P}$, Griffiths $\mathrm{E}$ et al. Immune responses in humans and animals to meningococcal transferrinbinding proteins: implications for vaccine design. Infect Immun 1994; 62: 2984-2990.

11. Rokbi B, Mazarin V, Maitre-Wilmotte G, Quentin-Millet M-J. Identification of two major families of transferrin receptors among Neisseria meningitidis strains based on antigenic and genomic features. FEMS Microbiol Lett 1993; 110: 51-58.

12. Griffiths E, Stevenson P, Ray A. Antigenic and molecular heterogeneity of the transferrin binding protein of Neisseria meningitidis. FEMS Microbiol Lett 1990; 69: 31-36.

13. Blanton KJ, Biswas GD, Tsai $\mathrm{J}$ et al. Genetic evidence that Neisseria gonorrhoeae produces specific receptors for transferrin and lactoferrin. $J$ Bacteriol 1990; 172: 5225-5235.

14. Cornelissen CN, Sparling PF. Binding and surface exposure characteristics of the gonococcal transferrin receptor are dependent on both transferrin-binding proteins. $J$ Bacteriol 1996; 178: $1437-1444$.

15. Irwin SW, Averil N, Cheng CY, Schryvers AB. Preparation and analysis of isogenic mutants in the transferrin receptor protein genes, $t b p \mathrm{~A}$ and $t b p \mathrm{~B}$, from Neisseria meningitidis. Mol Microbiol 1993; 8: 1125-1133.

16. Pintor M, Ferron L, Gómez JA, Gorringe A, Criado MT, Ferreirós CM. Blocking of iron uptake by monoclonal antibodies specific for the Neisseria meningitidis transferrinbinding protein 2. J Med Microbiol 1996; 45: 252-257.
17. Cornelissen $\mathrm{CN}$, Sparling PF. Iron piracy: acquisition of transferrin-bound iron by bacterial pathogens. Mol Microbiol 1994; 14: $843-850$

18. Yu RH, Schryvers AB. The interaction between human transferrin and transferrin binding protein 2 from Moraxella (Bramhamella) catarrhalis differs from that of other human pathogens. Microb Pathog 1993; 15: 433-445.

19. Irwin SW, Yu RH, Alcantara J, Schryvers AB. Transferrin and lactoferrin receptor proteins of Neisseria meningitidis. In: Condez-Glez CJ, Morse S, Rice P, Sparling PF, Calderon E (eds) Pathobiology and immunology of Neisseriaceae. Mexico, Publications Office of the National Institute of Public Health, Mexico, 1992: 392-397.

20. Williams P, Griffiths E. Bacterial transferrin receptors structure, function and contribution to virulence. Med Microbiol Immunol 1992; 181: 301-322.

21. Anderson JE, Sparling PF, Cornelissen CN. Gonococcal transferrin-binding protein 2 facilitates but is not essential for transferrin utilization. J Bacteriol 1994; 176: 3162-3170.

22. Cornelissen CN, Biswas GD, Sparling PF. Expression of gonococcal transferrin-binding protein 1 causes Escherichia coli to bind human transferrin. $J$ Bacteriol 1993; 175: 2448-2450.

23. Cornellisen CN, Biswas GD, Tsai J, Paruchuri DK, Thompson SA, Sparling PF. Gonococcal transferrin-binding protein 1 is required for transferrin utilization and is homologous to TonBdependent outer membrane receptors. $J$ Bacteriol 1992; 174: 5788-5797.

24. Pintor M, Ferron L, Gonez JA et al. Blocking of iron uptake from transferrin by antibodies against the transferrin binding proteins in Neisseria meningitidis. Microb Pathog 1996; 20 127-139. 